PROCEEDINGS OF THE

AMERICAN MATHEMATICAL SOCIETY

Volume 138, Number 8, August 2010, Pages 2973-2978

S 0002-9939(10)10413-4

Article electronically published on March 23, 2010

\title{
SMOOTHABILITY OF $\mathbb{Z} \times \mathbb{Z}$-ACTIONS ON 4-MANIFOLDS
}

\author{
NOBUHIRO NAKAMURA
}

(Communicated by Daniel Ruberman)

\begin{abstract}
We construct a nonsmoothable $\mathbb{Z} \times \mathbb{Z}$-action on the connected sum of an Enriques surface and $S^{2} \times S^{2}$, such that each of the generators is smoothable. We also construct a nonsmoothable self-homeomorphism on an Enriques surface.
\end{abstract}

\section{INTRODUCTION}

The purpose of this paper is to prove the existence of a nonsmoothable $\mathbb{Z} \times \mathbb{Z}$ action on a 4-manifold, such that each of the generators is smoothable:

Theorem 1.1. Let $X$ be the connected sum of an Enriques surface with $S^{2} \times$ $S^{2}$. Then, there exists a pair $\left(f_{1}, f_{2}\right)$ of self-homeomorphisms of $X$ which has the following properties:

(1) $f_{1}$ and $f_{2}$ commute.

(2) Each one of $f_{1}$ and $f_{2}$ can be smoothed for some smooth structure on $X$.

However, $f_{1}$ and $f_{2}$ cannot be smoothed at the same time for any smooth structure on $X$.

We also construct a nonsmoothable self-homeomorphism of an Enriques surface.

Theorem 1.2. There exists a self-homeomorphism of an Enriques surface $Y$ which is nonsmoothable with respect to any smooth structure on $Y$.

To prove these results, we modify the argument in [5] which analyzes the SeibergWitten moduli for families, and we give more convenient constraints on diffeomorphisms of 4-manifolds and then construct homeomorphisms which violate the constraints.

\section{CONSTRAints ON DIFFEOMORPHISMS}

In this section, we review the paper [5] and give some modifications of its results. In the paper [5], the author investigated the Seiberg-Witten moduli of families of 4-manifolds, and as an application, gave some constraints on diffeomorphisms of 4-manifolds. (We refer to the papers [7, 8] by D. Ruberman for other applications of the Seiberg-Witten theory on families to diffeomorphisms.) Let $X$ be a closed oriented smooth 4-manifold, and let $B$ be another closed manifold. We assume that a family $\mathbb{X}$ of $X$ over $B$ is given as a fiber bundle over $B$ whose fibers are

Received by the editors February 23, 2009 and, in revised form, November 22, 2009.

2010 Mathematics Subject Classification. Primary 57S05; Secondary 57M60, 57R57.

Key words and phrases. Group action, smoothability, Enriques surface.

(C)2010 American Mathematical Society 
diffeomorphic to $X$ as oriented manifolds. The fiber over $b \in B$ is denoted by $X_{b}$. Let $T(\mathbb{X} / B)$ be the tangent bundle along the fiber of $\mathbb{X}$, and assume a metric on $T(\mathbb{X} / B)$ is given. In order to consider the Seiberg-Witten equations on the family $\mathbb{X}$, we need a family of $\operatorname{Spin}^{c}$-structures on $\mathbb{X}$. One can obtain such a family of $\operatorname{Spin}^{c}$-structures if a $\operatorname{Spin}^{c}$-structure on $T(\mathbb{X} / B)$ is given. For this purpose, we gave somewhat complicated sufficient conditions. (See Proposition 2.1 of [5] and its correction [6].) In order to obtain a more convenient condition, we will take an alternative approach using classifying maps as described in 9 .

Let $\operatorname{Diff}(X)$ be the group of orientation-preserving diffeomorphisms of $X$. The classifying space $B \operatorname{Diff}(X)$ classifies families $\mathbb{X} \rightarrow B$ as above. Suppose a $\operatorname{Spin}^{c}$ structure $c$ on $X$ is given. Let us consider the group $\mathcal{S}(X, c)$ of pairs $(f, u)$, where $f$ is an orientation-preserving diffeomorphism satisfying $f^{*} c \cong c$, and $u: f^{*} c \rightarrow c$ is an isomorphism. The corresponding classifying space $B \mathcal{S}(X, c)$ classifies families $\mathbb{X} \rightarrow B$ with a $\operatorname{Spin}^{c}$-structure $\tilde{c}$ on $T(\mathbb{X} / B)$ such that the restriction of $\tilde{c}$ to each fiber is isomorphic to $c$. We have the forgetful map $\Phi: \mathcal{S}(X, c) \rightarrow \operatorname{Diff}(X)$. In general, $\Phi$ is not surjective. Let $\mathcal{N}(X, c)$ be the image of $\Phi$. Then there is an exact sequence

$$
1 \rightarrow \mathcal{G} \rightarrow \mathcal{S}(X, c) \rightarrow \mathcal{N}(X, c) \rightarrow 1,
$$

where $\mathcal{G}=\operatorname{Aut}(c) \cong \operatorname{Map}\left(X, S^{1}\right)$. Note that $B \mathcal{N}(X, c)$ classifies families $\mathbb{X} \rightarrow B$ whose structure groups are included in $\mathcal{N}(X, c)$. The exact sequence leads to a fibration

$$
B \mathcal{G} \rightarrow B \mathcal{S}(X, c) \rightarrow B \mathcal{N}(X, c) .
$$

Suppose a family $\mathbb{X} \rightarrow B$ classified by $\rho: B \rightarrow B \mathcal{N}(X, c)$ is given. If $b_{1}(X)=0$, then $B \mathcal{G}$ is homotopic to $\mathbb{C P}^{\infty} \cong B S^{1}$. In such a case, there is the sole obstruction to lift $\rho: B \rightarrow B \mathcal{N}(X, c)$ to $\tilde{\rho}: B \rightarrow B \mathcal{S}(X, c)$ in $H^{3}(B ; \mathbb{Z})$. In particular, if $\operatorname{dim} B \leq 2$, then every $\rho: B \rightarrow B \mathcal{N}(X, c)$ has a lift $\tilde{\rho}: B \rightarrow B \mathcal{S}(X, c)$.

Two kinds of families whose structure groups are in $\mathcal{N}(X, c)$ will be used in the proofs of the propositions below. The first is a mapping torus $X_{f}=(X \times$ $[0,1]) / f \rightarrow S^{1}$ defined by a diffeomorphism $f: X \rightarrow X$ satisfying $f^{*} c \cong c$. The second is a "double" mapping torus $X_{\left(f_{1}, f_{2}\right)} \rightarrow S^{1} \times S^{1}$ defined by two commutative diffeomorphisms $f_{1}$ and $f_{2}$ satisfying $f_{1}^{*} c \cong f_{2}^{*} c \cong c$. If the family $\mathbb{X}$ is $X_{f}$ or $X_{\left(f_{1}, f_{2}\right)}$ as above, we always have a $\operatorname{Spin}^{c}$-structure on $T(\mathbb{X} / B)$ by the previous paragraph.

When a $\operatorname{Spin}^{c}$-structure $\tilde{c}$ on $T(\mathbb{X} / B)$ is given, the Seiberg-Witten moduli space for the family $\mathbb{X}$ is given as follows. Let us define the bundle of parameters $\Pi \rightarrow B$ by

$$
\Pi=\left\{\left(g_{b}, \mu_{b}\right) \in \operatorname{Met}\left(X_{b}\right) \times \Omega^{2}\left(X_{b}\right) \mid *_{b} \mu_{b}=\mu_{b}\right\},
$$

where $\operatorname{Met}\left(X_{b}\right)$ is the space of Riemannian metrics on $X_{b}$ and $*_{b}$ is the Hodge star for the metric $g_{b}$. If we choose a section $\eta$ of $\Pi$, then the moduli space for the family $(\mathbb{X}, \tilde{c})$ is defined by

$$
\mathcal{M}(\mathbb{X}, \tilde{c}, \eta)=\coprod_{b \in B} \mathcal{M}\left(X_{b}, c_{b}, \eta_{b}\right),
$$

where $\mathcal{M}\left(X_{b}, c_{b}, \eta_{b}\right)$ is the Seiberg-Witten moduli space of the fiber $X_{b}$ with the Spin ${ }^{c}$-structure $c_{b}=\left.\tilde{c}\right|_{X_{b}}$ for the parameter $\eta_{b}=\left(g_{b}, \mu_{b}\right)$. Let us define the number $d(c)$ by

$$
d(c)=\frac{1}{4}\left(c_{1}(L)^{2}-\operatorname{sign}(X)\right)-\left(1+b_{+}\right),
$$


where $L$ is the determinant line bundle of $c$. Then, the virtual dimension of $\mathcal{M}(\mathbb{X}, \tilde{c}, \eta)$ is given by $d(c)+\operatorname{dim} B$.

In the propositions below, reducibles will play a special role. To see how many reducibles appear in $\mathcal{M}(\mathbb{X}, \tilde{c}, \eta)$, let us introduce a vector bundle $H_{\eta}^{+} \rightarrow B$ by $H_{\eta}^{+}=\coprod_{b \in B} H_{g_{b}}^{+}$, where $H_{g_{b}}^{+}$is the space of $g_{b}$-self-dual harmonic 2-forms of $X_{b}$. When $b_{1}=0, b_{+}=\operatorname{dim} B$ and $d(c)+\operatorname{dim} B=1$, it is proved that the number of reducibles in $\mathcal{M}(\mathbb{X}, \tilde{c}, \eta)$ is equal modulo 2 to the number of zeros of a generic section of $H_{\eta}^{+}$.

With these understood, we can modify the results in [5] as follows.

Proposition 2.1. Let $X$ be a closed oriented smooth 4-manifold with $b_{1}=0$ and $b_{+}=1, c$ a Spin $^{c}$-structure on $X$ with $d(c)=0$, and $f: X \rightarrow X$ an orientationpreserving diffeomorphism. If $f^{*} c$ is isomorphic to $c$, then $f$ preserves the orientation of $H^{+}(X ; \mathbb{R})$.

The proof of Proposition 2.1 is given by a slight modification of the proof of Theorem 1.2 of [5]. The outline is as follows. Suppose a diffeomorphism $f$ satisfying $f^{*} c \cong c$ is given and consider the mapping torus $X_{f} \rightarrow B=S^{1}$ by $f$. Under the assumptions of Proposition 2.1, the moduli space $\mathcal{M}\left(X_{f}, \tilde{c}, \eta\right)$ of $X_{f}$ for a generic choice of $\eta$ is a compact 1-dimensional manifold whose boundary points are reducibles. If $f$ reverses the orientation of $H^{+}(X ; \mathbb{R})$, then $H_{\eta}^{+}$is a nontrivial real line bundle over $S^{1}$. Hence, the number of reducibles should be odd. However, this is a contradiction, because the number of boundary points of a compact 1-dimensional manifold is even.

Similarly, we can prove the following by modifying the proof of Theorem 1.1 of [5]:

Proposition 2.2. Let $X$ be a closed oriented smooth 4-manifold with $b_{1}=0$ and $b_{+}=2$, and $c$ a Spin ${ }^{c}$-structure on $X$ with $d(c)=-1$. Suppose a pair $\left(f_{1}, f_{2}\right)$ of orientation-preserving diffeomorphisms on $X$ satisfies the following conditions:

(1) $f_{1}$ and $f_{2}$ commute.

(2) $f_{1}$ and $f_{2}$ preserve the isomorphism class of $c$.

Then, $w_{2}\left(H_{\eta}^{+}\right)=0$, where $H_{\eta}^{+}$is the bundle associated to the family $X_{\left(f_{1}, f_{2}\right)}$.

\section{Nonsmoothable SELF-Homeomorphism on EnRIQUes Surface}

The purpose of this section is to prove Theorem 1.2, First, note that the Enriques surface can be decomposed into three connected summands topologically by a theorem due to Hambleton and Kreck [3. In fact, the following theorem can be proved from Theorem 3 in [3] and its proof.

Theorem 3.1 (Hambleton-Kreck [3]). The Enriques surface is homeomorphic to a topological manifold $Y=\left|E_{8}\right| \# \Sigma \#\left(S^{2} \times S^{2}\right)$, where $\left|E_{8}\right|$ is the " $E_{8}$-manifold", i.e., the simply connected closed topological 4-manifold whose intersection form is the negative definite $E_{8}$, and $\Sigma$ is a nonspin rational homology 4-sphere with fundamental group $\mathbb{Z} / 2$.

Remark 3.2. Neither $\Sigma$ nor $\left|E_{8}\right| \#\left(S^{2} \times S^{2}\right)$ is smoothable, because both have nontrivial Kirby-Siebenmann invariants.

Now, we will construct a self-homeomorphism of $Y$. Let $\varphi: S^{2} \times S^{2} \rightarrow S^{2} \times S^{2}$ be an orientation-preserving diffeomorphism which has the following properties: 
(1) There is a 4-ball $B_{0} \subset S^{2} \times S^{2}$ such that the restriction of $\varphi$ to $B_{0}$ is the identity map on $B_{0}$.

(2) $\varphi$ reverses the orientation of $H^{+}\left(S^{2} \times S^{2} ; \mathbb{R}\right)$.

Such a $\varphi$ can be easily constructed as follows:

Example 3.3. Assume $S^{2} \times S^{2}=\mathbb{C P}^{1} \times \mathbb{C P}^{1}$. Let $\varphi_{0}$ be the automorphism on $\mathbb{C P}^{1} \times \mathbb{C P}^{1}$ defined by complex conjugation. Choose a fixed point $p_{0}$ of $\varphi_{0}$. Then, a required $\varphi$ is obtained by perturbing $\varphi_{0}$ around $p_{0}$ to be the identity on a neighborhood of $p_{0}$.

Let us define a self-homeomorphism $f$ on $Y$ by $f=\operatorname{id}_{\left|E_{8}\right| \# \Sigma} \# \varphi$, where $\operatorname{id}_{\left|E_{8}\right| \# \Sigma}$ is the identity map of $\left|E_{8}\right| \# \Sigma$. (Note that we can take a connected sum of $\varphi$ with $\operatorname{id}_{\left|E_{8}\right| \# \Sigma}$ on $B_{0} \subset S^{2} \times S^{2}$.) Now, we claim that $f$ is nonsmoothable with respect to any smooth structure on $Y$.

To prove that $f$ is nonsmoothable, we will temporarily need a topological $\mathrm{Spin}^{c}$ structure on the topological manifold $Y$. Let us make a digression for it. (A brief description for topological spin structures is found in [1, Section 3. See also 2, 10.2B.) By Kister-Mazur's theorem, the tangent microbundle $\tau Y$ of $Y$ determines up to isomorphism the topological "frame" bundle $F$ whose structure group is contained in STop(4), the group of orientation-preserving homeomorphisms of $\mathbb{R}^{4}$ preserving the origin. It is known that the inclusion $\mathrm{SO}(4) \rightarrow \operatorname{STop}(4)$ induces an isomorphism on $\pi_{1}$ and both have trivial $\pi_{0}$ and $\pi_{2}$ (4, $\mathrm{V}$ and [2], 8.7). Let $\phi: \operatorname{SpinTop}(4) \rightarrow \operatorname{STop}(4)$ be the unique double covering. Then, a topological spin structure on $Y$ is defined as a double covering $\tilde{F} \rightarrow F$ whose restriction to each fiber is $\phi$. Topological $\mathrm{Spin}^{c}$-structures are similarly defined by using $\operatorname{SpinTop}^{c}(4):=\operatorname{SpinTop}(4) \times_{\mathbb{Z}_{2}} \mathrm{U}(1) \rightarrow \operatorname{STop}(4)$. The set of isomorphic classes of topological Spin ${ }^{c}$-structures has a principal action of $H^{2}(Y ; \mathbb{Z})$ as in the case of true $\operatorname{Spin}^{c}$-structures.

Lemma 3.4. Let $c$ be the topological $\operatorname{Spin}^{c}$-structure on $Y$ whose $c_{1}(L)$ is a torsion class. Then $f^{*} c$ is isomorphic to $c$.

Proof. In this proof, all spin/Spin ${ }^{c}$-structures are understood as topological ones. The $\operatorname{Spin}^{c}$-structure $c$ can be identified with the sum of the unique spin structure $c_{0}$ on $\left|E_{8}\right| \#\left(S^{2} \times S^{2}\right)$ and a Spin ${ }^{c}$-structure $c_{\Sigma}$ on $\Sigma$ whose $c_{1}(L)$ is a torsion class. Since $f$ is the identity on $\Sigma, f$ preserves $c_{\Sigma}$. On the other hand, since $c_{0}$ is the unique spin structure on $\left|E_{8}\right| \#\left(S^{2} \times S^{2}\right), f^{*} c_{0} \cong c_{0}$.

Let us prove that $f$ is nonsmoothable. Once a smooth structure on $Y$ is given, we have a reduction of the topological frame bundle $F$ to the true frame $\mathrm{SO}(4)$-bundle, and also a topological Spin ${ }^{c}$-structure is reduced to the corresponding true $\mathrm{Spin}^{c}$ structure. Suppose $f$ is smoothed. By Lemma 3.4 $f^{*} c$ is isomorphic to $c$ as true Spin $^{c}$-structures. On the other hand, $f$ is an orientation-preserving diffeomorphism which reverses the orientation of $H^{+}(Y)$. This contradicts Proposition 2.1.

\section{Proof of Theorem 1.1}

In this section, we prove Theorem 1.1 To begin with, we collect the ingredients needed for our construction. Let $S_{0}=S^{2} \times S^{2}$ and fix a 4-ball $B_{0}^{\prime} \subset S_{0}$. For $i=1,2$, let $\left(S_{i}, \varphi_{i}\right)$ be copies of $\left(S^{2} \times S^{2}, \varphi\right)$ and fix smooth 4-balls $B_{i}^{\prime} \subset S_{i}$ on which $\left.\varphi_{i}\right|_{B_{i}^{\prime}}$ are the identity maps. If we make a connected sum of $S_{i}(i=0,1,2)$ with another 
manifold, remove $B_{i}^{\prime}$ from $S_{i}$ and glue it along the boundary to another. Let $Z$ be $\left|E_{8}\right| \# \Sigma$. Later, we will choose 4-balls $B_{0}, B_{1}$ and $B_{2}$ in $Z$ so that

- $B_{1} \cap B_{0}=\emptyset, B_{1} \cap B_{2}=\emptyset$, and

- if we make a connected sum of $Z$ with $S_{i}(i=0,1,2)$, remove $B_{i}$ from $Z$ and glue $\overline{Z \backslash B_{i}}$ and $\overline{S_{i} \backslash B_{i}^{\prime}}$. (The resulting connected sum will be denoted as $Z \# \partial B_{i} S_{i}$.)

Let $E_{1}$ and $E_{2}$ be smooth 4-manifolds homeomorphic to an Enriques surface. The basic idea of our construction is as follows. The connected sum $S_{1} \#{ }_{\partial B_{1}} Z Z_{\partial B_{2}} S_{2}$ can be assumed as a connected sum of an Enriques surface with $S^{2} \times S^{2}$ in two ways: $S_{1} \# E_{1}$ and $E_{2} \# S_{2}$. Then, two commutative homeomorphisms $f_{1}, f_{2}$ will be defined by $\varphi_{1} \# \operatorname{id}_{E_{1}}$ and $\operatorname{id}_{E_{2}} \# \varphi_{2}$,

Let us begin the precise construction. Choose a 4-ball $B_{0} \subset Z$ arbitrarily. Then $Z \#_{\partial B_{0}} S_{0}$ is homeomorphic to an Enriques surface. Fix a homeomorphism $\bar{h}_{1}: E_{1} \rightarrow Z \#{ }_{\partial B_{0}} S_{0}$. Next, choose $B_{1}$ so that $D_{1}:=\bar{h}_{1}^{-1}\left(B_{1}\right)$ is a smoothly embedded 4-ball in $E_{1}$. Take a smooth connected sum $S_{1} \#_{\partial D_{1}} E_{1}$ and a (topological) connected sum $S_{1} \# \partial B_{1} Z \#{ }_{\partial B_{0}} S_{0}$ so that a homeomorphism $h_{1}=\operatorname{id}_{S_{1}} \# \bar{h}_{1}: S_{1} \# \partial D_{1} E_{1}$ $\rightarrow S_{1} \#{\partial B_{1}} Z \#_{\partial B_{0}} S_{0}$ is defined.

Note that $S_{1} \#{ }_{\partial B_{1}} Z$ is also homeomorphic to an Enriques surface. Fix a homeomorphism $\bar{h}_{2}: E_{2} \rightarrow S_{1} \#_{\partial B_{1}} Z$. Choose $B_{2}$ so that $D_{2}:=\bar{h}_{2}^{-1}\left(B_{2}\right)$ is a smoothly embedded 4-ball in $E_{2}$. Take a smooth connected sum $E_{2} \# \partial D_{2} S_{2}$ and a (topological) connected sum $S_{1} \#{ }_{\partial B_{1}} Z \#_{\partial B_{2}} S_{2}$ so that a homeomorphism $h_{2}=\bar{h}_{2} \# \operatorname{id}_{S_{2}}$ : $E_{2} \# \partial D_{2} S_{2} \rightarrow S_{1} \#{\partial B_{1}} Z \#_{\partial B_{2}} S_{2}$ is defined.

Define the self-diffeomorphism $\bar{f}_{1}$ on $S_{1} \# \partial D_{1} E_{1}$ by $\bar{f}_{1}=\varphi_{1} \# \operatorname{id}_{E_{1}}$, and $\bar{f}_{2}$ on $E_{2} \# \partial D_{2} S_{2}$ by $\bar{f}_{2}=\operatorname{id}_{E_{2}} \# \varphi_{2}$. Choose a homeomorphism $h: S_{1} \# \partial B_{1} Z \# \partial B_{2} S_{2} \rightarrow$ $S_{1} \#{ }_{\partial B_{1}} Z \#{ }_{\partial B_{0}} S_{0}$ so that $\left.h\right|_{S_{1} \backslash B_{1}^{\prime}}$ is the identity map. Via homeomorphisms $h$, $h_{1}$ and $h_{2}$, we obtain self-homeomorphisms $f_{1}$ and $f_{2}$ of $X:=S_{1} \#_{\partial B_{1}} Z \#_{\partial B_{2}} S_{2}$ induced from $\bar{f}_{1}$ and $\bar{f}_{2}$, respectively. Then each $f_{i}(i=1,2)$ is smoothable for the smooth structure $E_{i} \# \partial D_{i} S_{i}$. Clearly, $f_{1}$ and $f_{2}$ commute. Let $c$ be the $\operatorname{Spin}^{c}$ structure on $X$ whose $c_{1}(L)$ is a torsion class. As in Lemma 3.4 we can see that $f_{1}$ and $f_{2}$ preserve the isomorphism class of $c$. However, $w_{2}\left(H_{\eta}^{+}\right) \neq 0$ by construction. By Proposition 2.2 $f_{1}$ and $f_{2}$ cannot be smoothed at the same time. Thus, Theorem 1.1 is proved.

\section{REMARKS}

We give two remarks. The first is on another possibility for an application of Proposition 2.2. The following problem would be interesting: Find two diffeomorphisms of a smooth manifold homeomorphic to a connected sum of an Enriques surface $E$ with $S^{2} \times S^{2}$ that are simultaneously smoothable, commute up to isotopy, but do not have representatives in their isotopy classes that commute.

The second remark is on a generalization of the construction of the moduli spaces for families. In fact, we can construct the moduli space for a family without a family of Spin ${ }^{c}$-structures. More precisely, we claim the following: When a family $\mathbb{X} \rightarrow B$ is classified by $\rho: B \rightarrow B \mathcal{N}(X, c)$, we can always construct the moduli space $\mathcal{M}(\mathbb{X}, c)$ for the family $\mathbb{X}$, even if $\rho$ does not have a lift $\tilde{\rho}: B \rightarrow B \mathcal{S}(X, c)$. The construction is outlined as follows. By taking local trivializations, the family $\mathbb{X}$ can be given via transition functions $\psi_{\beta \alpha}: U_{\alpha} \cap U_{\beta} \rightarrow \mathcal{N}(X, c)$ for an appropriate covering $\left\{U_{\lambda}\right\}_{\lambda \in \Lambda}$ of $X$. Suppose the intersection of every two members in $\left\{U_{\lambda}\right\}_{\lambda \in \Lambda}$ is contractible. Then we can take a lift of each $\psi_{\beta \alpha}: U_{\alpha} \cap U_{\beta} \rightarrow \mathcal{N}(X, c)$ to $\tilde{\psi}_{\beta \alpha}: U_{\alpha} \cap U_{\beta} \rightarrow \mathcal{S}(X, c)$. 
In general, such $\tilde{\psi}_{\beta \alpha}$ do not satisfy the cocycle condition, but satisfy it up to gauge; i.e., $\psi_{\gamma \beta} \psi_{\beta \alpha} \psi_{\gamma \alpha}^{-1}$ is a gauge transformation. One can define local families $\mathcal{M}\left(U_{\lambda} \times X, c\right)=\coprod_{b \in U_{\lambda}} \mathcal{M}(\{b\} \times X, c) \rightarrow U_{\lambda}$ of moduli spaces and attaching maps $\tilde{\psi}_{\beta \alpha}^{*}$ between them induced from $\tilde{\psi}_{\beta \alpha}$. (Here, we need a little care on metrics and perturbations.) Since the moduli spaces are defined as the quotient spaces divided by the gauge transformations, $\tilde{\psi}_{\beta \alpha}^{*}$ satisfy the cocycle condition. Therefore, the global family $\mathcal{M}(\mathbb{X}, c)$ can be constructed from the local families $\mathcal{M}\left(U_{\lambda} \times X, c\right)$ via $\tilde{\psi}_{\beta \alpha}^{*}$. Such a family $\mathcal{M}(\mathbb{X}, c)$ would be useful for further applications.

\section{ACKNOWLEDGEMENTS}

The author would like to thank M. Furuta, Y. Kametani, K. Kiyono and M. Ue for helpful discussions and comments on earlier versions of the paper. It is also his pleasure to thank the referee for detailed comments and many valuable suggestions which enabled him to correct and improve the paper.

\section{REFERENCES}

[1] A. Edmonds, Aspects of group actions on four-manifolds, Topology Appl. 31 (1989), no. 2, 109-124. MR.994404 (90h:57050)

[2] M. H. Freedman and F. Quinn, Topology of 4-manifolds, Princeton Mathematical Series, 39. Princeton University Press, Princeton, NJ, 1990. MR 1201584 (94b:57021)

[3] I. Hambleton and M. Kreck, Smooth structures on algebraic surfaces with cyclic fundamental group, Invent. Math. 91 (1988), no. 1, 53-59. MR918236 (89a:57043)

[4] R. C. Kirby and L. C. Siebenmann, Foundational essays on topological manifolds, smoothings, and triangulations. Annals of Mathematics Studies, No. 88. Princeton University Press, Princeton, NJ; University of Tokyo Press, Tokyo, 1977. MR.0645390 (58:31082)

[5] N. Nakamura, The Seiberg-Witten equations for families and diffeomorphisms of 4-manifolds, Asian J. Math. 7 (2003), no. 1, 133-138. MR2015245 (2004h:57037)

[6] Correction, Asian J. Math. 9 (2005), no. 2, 185. MR2176601(2006e:57040)

[7] D. Ruberman, An obstruction to smooth isotopy in dimension 4, Math. Res. Lett. 5 (1998), no. 6, 743-758. MR.1671187 (2000c:57061)

[8] D. Ruberman, Positive scalar curvature, diffeomorphisms and the Seiberg-Witten invariants, Geom. Topol. 5 (2001), 895-924. MR1874146 (2002k:57076)

[9] M. Szymik, Characteristic cohomotopy classes for families of 4-manifolds, preprint.

Graduate School of Mathematical Sciences, University of Tokyo, 3-8-1, Komaba, MEgurO-KU, TOKYO, 153-8914, JAPAN

E-mail address: nobuhiro@ms.u-tokyo.ac.jp 